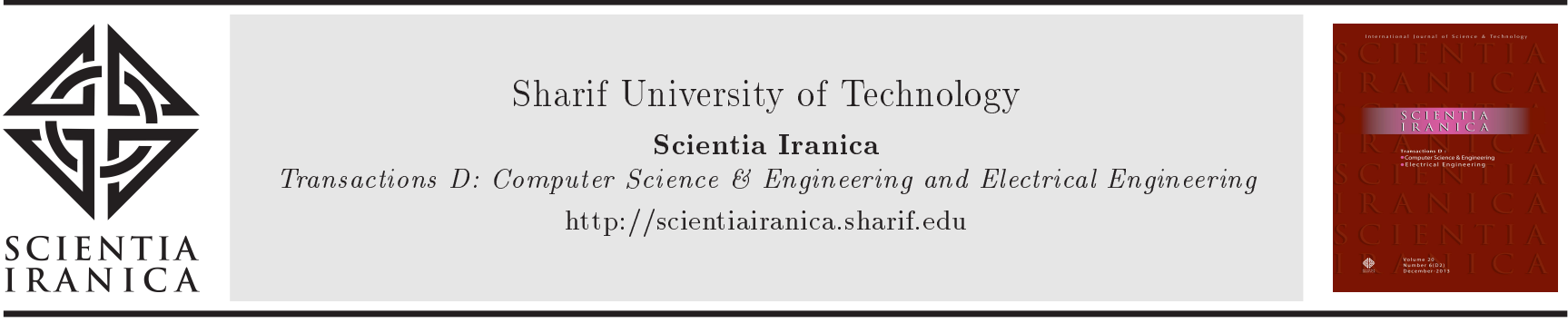

\title{
A new modulation scheme in polymer optical fiber communications using Jacket matrix spreading
}

\author{
P. Miao ${ }^{\mathrm{a}, \mathrm{b}, *}, \mathrm{~L} . \mathrm{Wu}^{\mathrm{b}}$, and L. Peng ${ }^{\mathrm{b}}$ \\ a. School of Electronic and Information Engineering, Qingdao University, Ningxia Road, Qingdao, 266071, China. \\ b. School of Information Science and Engineering, Southeast University, 2 Sipaolou, Nanjing, 210096, China. \\ Received 2 March 2015; received in revised form 7 May 2017; accepted 17 June 2017
}

\section{KEYWORDS \\ Polymer optical fiber; Peak to average power ratio; \\ Modulation scheme; Jacket matrix spreading.}

\begin{abstract}
In this study, a novel DMT scheme based on Jacket matrix Spreading (JS) is proposed to mitigate the high Peak-to-Average Power Ratio (PAPR) of Discrete MultiTone (DMT) modulation in Polymer Optical Fibers (POF) which serve communication systems. Approximations of PAPR distribution for DMT signals are presented accurately. Single-band JS-DMT and Multi-Band (MB)-JS-DMT are introduced in POF transmission link for PAPR reduction. Offline processing and simulations are adopted to investigate the performance of these new schemes with regard to the PAPR reduction, Power Spectral Density (PSD), data rate, and Bit Error Rate (BER). The results demonstrate that, by applying the JS methodology, up to $4 \mathrm{~dB}$ PAPR reduction is achieved. JS-DMT is sensitive to the bandwidth range, whereas MB-JS-DMT owns the robustness ability against fiber nonlinearity. Compared with some well-known PAPR reduction techniques, the proposed schemes can be easily applied to the DMT modem and achieve the PAPR reduction efficiently without degrading the BER, data rate, and PSD performance, demonstrating the feasibility and validity of these two methods in POF transmission.

(C) 2019 Sharif University of Technology. All rights reserved.
\end{abstract}

\section{Introduction}

Large core diameter Step-Index Polymer Optical Fibers (SI-POFs) are proposed as a preferred alternative transmission medium for high-speed short-range data communications and have attracted significant research interest $[1,2]$. Most recent works have already shown experimentally that adopting spectrally efficient modulation techniques within Intensity Modulation with Direct Detection (IM/DD) structures could overcome the bandwidth limitation of the SI-POF and extend the system capacity [3]. With the low-cost consideration, Discrete Multi-Tone (DMT) modulation [4-7], demonstrating strong ability of anti-multipath and anti-

\footnotetext{
*. Corresponding author. Tel.: +86-182-6623-6416
} E-mail address: miaopusias@126.com (P. Miao) fading, is regarded to be future-proof solutions for maximizing the transmission rate in SI-POF system $[8,9]$. Therefore, employing the DMT with bit-loading techniques makes the Gigabit transmission over SI-POF available. However, there are still some challenging design issues. One of the major problems is high Peak-to-Average Power Ratio (PAPR) of the DMT signals [10]. When the DMT signals with high PAPR are being transmitted over the POFs, distortion occurs and nonlinear effects increase, resulting in degradation of the overall performance. Therefore, adopting an appropriate method for reducing the PAPR should receive the utmost degree of attention.

Seeking a solution to reduce the high PAPR, extensive researches have been carried out and various PAPR reduction approaches have been proposed in wireless communication systems [11,12]. However, some of these common methods may not be directly used in POF systems. Clipping, filtering, and com- 
panding are types of famous signal distortion schemes that directly limit the peak envelope of the transmitting signal to a desired value [13-15]. However, the signal processing leads to in-band distortions and out-of-band high frequency components, which will further degrade the Bit Error Rate (BER) performance and reduce the spectral efficiency. Coding, selective mapping (SLM), and Partial Transmit Sequences (PTS) are the typical signal scrambling schemes [16,17] that statistically improve the characteristics of the PAPR distribution and provide pretty good PAPR reduction performance. However, they have very high computational complexity, and the side information, which contains the optimal phase factors, must be transmitted to the receiver for the data recovery. Besides, large amounts of hardware resources will be consumed and the BER may be degraded if the SI is not detected accurately. In addition, it is worth noting that the design of phase vectors for SLM and PTS is also troublesome. Therefore, signal distortion schemes and scrambling techniques are limited by the BER performance and the computational complexity.

In this paper, the Jacket matrix [18-20] Spreading (JS) in terms of JS-DMT and Multi-Band (MB)-JSDMT is proposed in POF system. The main goal is to focus on the application of JS technique in DMT modem for PAPR reduction. Extensive results and discussions show that these proposed schemes are able to achieve a better compromise with regard to the $\mathrm{PAPR}$ reduction, $\mathrm{BER}$, data rate and spectral efficiency.

The remainder of this paper is organized as follows. Section 2 outlines the PAPR of DMT and investigates its statistical distributions. Section 3 describes the correlation property between DMT symbols and peak powers. In Section 4, two kinds of DMT modem based on single-band and multi-band spreading are proposed in POF transmission. System performance comparisons regarding the PAPR reduction, BER and Power Spectral Density (PSD) are illustrated and discussed in Section 5. Section 6 presents the conclusion.

Unless otherwise mentioned, lower and upper case bold-face letters denote time-domain and frequencydomain vectors, respectively, e.g., $\mathbf{x}$ and $\mathbf{X} . x_{n}$ denotes the $(n+1)$ th element of column vector $\mathbf{x}$. Let $\mathbb{E}\{\cdot\},(\cdot)^{T}$, $(\cdot)^{*},|\cdot|, \mathbb{R}\{\cdot\}$, and $\mathcal{Z}(\cdot)$ denote the ensemble average, transpose, complex conjugate, absolute, real component, and symmetric conjugate operator, respectively. Let $\mathcal{N}\left(\mu, \sigma^{2}\right)$ be the Gaussian distribution with mean $\mu$ and variance $\sigma^{2}$.

\section{PAPR problem and statistical distribution}

There are $N$ sub-carriers for a DMT symbol; the bit stream is mapped onto $X_{u}=A_{u}+j B_{u}(u=1, \cdots, N-$
1) based on the Quadrature Amplitude Modulation (QAM) constellation, and then the DMT signals are illustrated as follows [4-7]:

$$
\begin{aligned}
& x_{k}=\frac{1}{\sqrt{2 N}} \sum_{n=0}^{2 N-1} C_{n} \exp \left(\frac{j 2 \pi n k}{2 N}\right), \\
& C_{2 N-u}=C_{u}^{*}=X_{u}^{*},
\end{aligned}
$$

where $x_{k}(k=0,1, \cdots, 2 N-1)$ is a real value which requires $2 N$ points of Inverse Fast Fourier Transform (IFFT) computation, $C_{k}$ follows Hermitian symmetry property given by Eq. (2), and $C_{0}=C_{N}=X_{0}=0$. We assume $X_{u}$ to be statistically independent and identically distributed. As $N$ is large enough, both $A_{u}$ and $B_{u}$ have a Gaussian distribution $\mathcal{N}\left(0, \frac{\sigma^{2}}{2}\right)$ due to the central limit theorem. After determining conjugate symmetry in Eq. (2), we can obtain $\mathbb{E}\left\{C_{k}\right\}=0$ and $\mathbb{E}\left\{\left|C_{k}\right|^{2}\right\}=\sigma^{2}$. Therefore, the real $x_{k}$ approaches $\mathcal{N}\left(0, \sigma^{2}\right)$.

The PAPR of the transmitted signal is defined as the ratio of the maximum peak power to the average power during a symbol period, expressed as follows $[21,22]$ :

$$
\mathrm{PAPR}_{d} \triangleq \frac{\max _{0 \leq k \leq 2 N-1}\left\{\left|x_{k}\right|^{2}\right\}}{\mathbb{E}\left\{\left|x_{k}\right|^{2}\right\}}=\frac{\max _{0 \leq k \leq 2 N-1}\left\{\left|x_{k}\right|^{2}\right\}}{\sigma^{2}}
$$

According to the central limit theorem, we can obtain:

$$
\begin{aligned}
& P_{r}\left(\frac{\left|x_{k}\right|^{2}}{\sigma^{2}} \leq r\right)=P_{r}\left(-\sqrt{r} \leq \frac{x_{k}}{\sigma} \leq \sqrt{r}\right) \\
& \quad \approx \operatorname{erf}\left(\sqrt{\frac{r}{2}}\right)
\end{aligned}
$$

where $\operatorname{erf}(\psi)=2 / \sqrt{\pi} \int_{0}^{\psi} e^{-t^{2}} d t, P_{r}(\cdot)$ is the probability, and $r$ is the threshold. Generally, the Complementary Cumulative Distribution Function (CCDF), which denotes the probability of the PAPR that exceeds a given threshold within one symbol duration, is used to evaluate the performance in PAPR reduction. Therefore, the CCDF can be calculated as follows:

$$
\mathrm{CCDF}_{d}=P_{r}\left\{\mathrm{PAPR}_{d}>r\right\}=1-e r f^{2 N}\left(\sqrt{\frac{r}{2}}\right) .
$$

Continuous-time base-band DMT signals can be approximately represented by $L_{0}$ times oversampled discrete time DMT signals. Thus, the IFFT outputs are illustrated as follows:

$$
\begin{aligned}
& x_{k_{0}}=\frac{1}{\sqrt{2 N L_{0}}} \sum_{n=0}^{2 N L_{0}-1} C_{n}^{\prime} \exp \left(\frac{j 2 \pi n k_{0}}{2 N L_{0}}\right), \\
& k_{0}=0,1, \cdots, 2 N L_{0}-1 .
\end{aligned}
$$


For $0 \leq n \leq\left(L_{0}-1\right) N-1, C_{n}^{\prime}$ is presented as follows:

$$
C_{n}^{\prime}= \begin{cases}X_{n}, & 0 \leq n \leq N-1 \\ 0, & N \leq n \leq\left(L_{0}-1\right) N-1\end{cases}
$$

For $\left(L_{0}-1\right) N+1 \leq n \leq 2 L_{0} N-1, C_{n}^{\prime}$ also follows the Hermitian symmetry property, and $C_{0}^{\prime}=C_{\left(L_{0}-1\right) N}^{\prime}=$ 0 . Therefore, as $N$ is sufficiently large, the real value $\left\{x_{k_{0}}, k_{0}=0,1, \cdots, 2 N L_{0}-1\right\}$ converges in distribution to $\{x(t), 0 \leq t \leq T\}$, where $T=\Delta f^{-1}$ is the symbol period and $f_{s}=2 N L_{0} \Delta f$ is the sampling rate. Thus, the PAPR of $x(t)$ can be written as follows:

$$
\operatorname{PAPR}_{c} \triangleq \frac{\max _{0 \leq t \leq T} x^{2}(t)}{\mathbb{E}\left\{|x(t)|^{2}\right\}}=\frac{\max _{0 \leq t \leq T} x^{2}(t)}{\sigma^{2}}
$$

Obviously, variable $v(t)=\frac{x(t)}{\sigma}$ converges to stationary $\mathcal{N}(0,1)$. According to the extreme value theory, the corresponding CCDF can be calculated by:

$$
\mathrm{CCDF}_{c}=P_{r}\left\{\mathrm{PAPR}_{c}>r\right\}=1-\exp \left(\frac{-2 N}{\sqrt{3}} e^{-\frac{r}{2}}\right)
$$

Therefore, stochastic characteristics of the PAPR for discrete-time $x_{k}$ and continuous-time $x(t)$ can be estimated by Eqs. (5) and (9), respectively.

It is shown in [11] that choosing $L_{0} \geq 4$ is sufficient to approximate the peak value of $x(t)$. Therefore, the sequences of $x_{k}$ and $x_{k_{0}}$ are obtained by $2 N$ point and $16 N$ point IFFT computations, respectively. Considering $N=64,128,256$, and 512, the theoretical approximations and computer simulations of PAPR in terms of $\mathrm{CCDF}_{d}$ and $\mathrm{CCDF}_{c}$ are shown in Figure 1, where the solid lines denote the simulation results and the dash lines represent the approximations. It is clearly manifested that the approximations agree well with the simulations and can predict the distribution of the PAPR for discrete-time and continuous-time DMT accurately. In addition, as $N$ increases, the PAPR problem deteriorates; thus, it should receive greater more attention.

\section{Correlation property}

Taking the example of the discrete-time DMT signal, the power of $x_{k}$ can be calculated by:

$$
\begin{aligned}
\left|x_{k}\right|^{2}= & \frac{1}{2 N} \sum_{p=0}^{2 N-1} \sum_{q=0}^{2 N-1} C_{p} C_{q}^{*} \exp \left(\frac{j 2 \pi(p-q) k}{2 N}\right)=\frac{1}{2} \\
& +\frac{1}{N} \mathbb{R}\left\{\sum_{q=1}^{2 N-1} \exp \left(\frac{j 2 \pi q k}{2 N}\right)^{2 N-1-q} \sum_{p=0}^{2} C_{p+q} C_{p}^{*}\right\}
\end{aligned}
$$

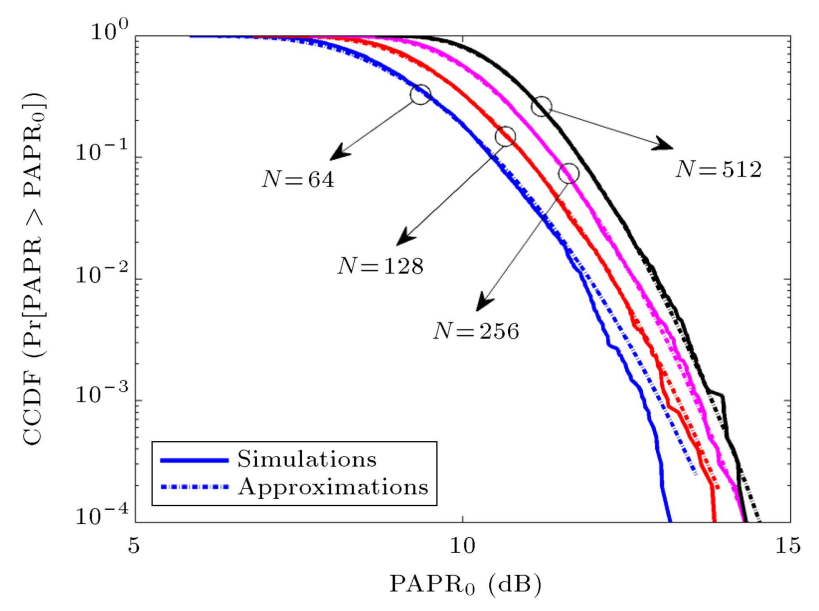

(a)

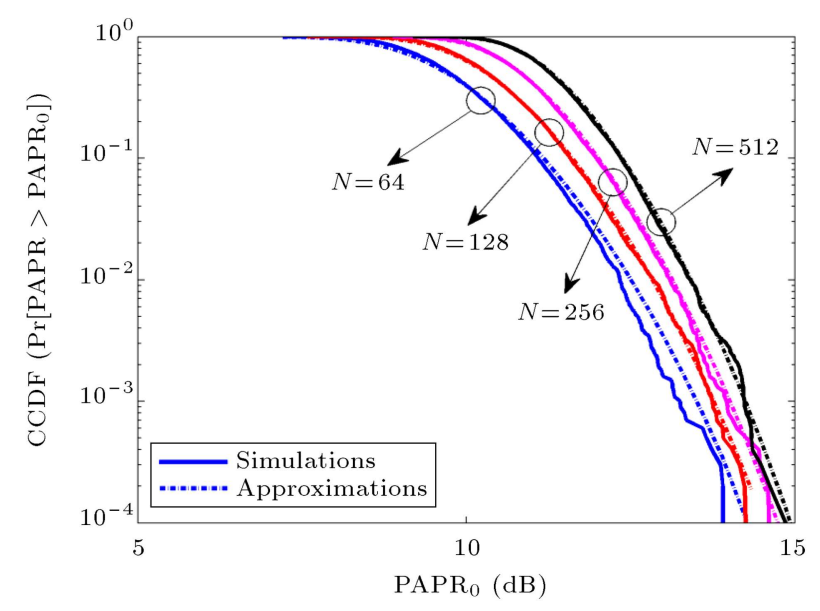

(b)

Figure 1. Comparison of approximations with simulations of PAPR: (a) Discrete time and (b) continuous time.

Defining $\rho_{C}(q)=\sum_{p=0}^{2 N-1-q} C_{p+q} C_{p}^{*}$, is the aperiodic autocorrelation function (ACF) of $C_{k}$. For an arbitrary complex $\mathcal{X}_{k}$, the following inequality holds: $\mathbb{R}\left\{\mathcal{X}_{k}\right\} \leq$ $\left|\mathcal{X}_{k}\right|$ and $\left|\sum_{k=0}^{N-1} \mathcal{X}_{k}\right| \leq \sum_{k=0}^{N-1}\left|\mathcal{X}_{k}\right|$. Thus, the PAPR with unit mean power of $\sigma^{2}=1$ satisfies:

$$
\begin{aligned}
\operatorname{PAPR} & =\frac{\max _{0 \leq k \leq 2 N-1}\left\{\left|x_{k}\right|^{2}\right\}}{\sigma^{2}} \leq \frac{1}{2}+\frac{1}{N} \sum_{q=1}^{2 N-1}\left|\rho_{C}(q)\right| \\
& =\frac{1}{2}+\frac{1}{N} \xi_{C}
\end{aligned}
$$

where $\xi_{C}=\sum_{q=1}^{2 N-1}\left|\rho_{C}(q)\right|$. When input symbols $C_{n}$, are equal to each other, $\max _{0 \leq k \leq 2 N-1}\left\{\left|x_{k}\right|^{2}\right\}$ within one symbol duration could reach the maximum value. Therefore, there is a close relationship between the PAPR and the correlation property of input $C_{n}$. If an appropriate method is performed to make input $C_{n}$ 
with lower $\xi_{C}$, then the transmitted signal with lower $\max _{0 \leq k \leq 2 N-1}\left\{\left|x_{k}\right|^{2}\right\}$ will be obtained, and the probability of the PAPR that exceeds a given threshold within one symbol duration is reduced, meaning that the PAPR reduction can be achieved consequently. Based on this principle, the novel DMT scheme will be proposed in the following to lower $\xi_{C}$, thus reducing the PAPR indirectly.

\section{The proposed scheme}

\subsection{Jacket matrix}

Derived and extended from the weighted Hadamard matrix, Jacket matrix is a class of matrix that is simple to calculate, easily element-wise invertible, and sizeflexible [18-20]. It is widely used in data compression, images processing, and orthogonal space-time coding. The entries of Hadamard matrix are +1 or -1 , whereas those of Jacket matrix are \pm 1 and $\pm i$. Assuming that $\mathbf{J}=\left(J_{m, l}\right)_{N}$ signifies an $N \times N$ Jacket matrix, the inverse matrix can be calculated by:

$$
\mathbf{J}^{+}=\frac{1}{N}\left(J_{m, l}^{-1}\right)_{N}^{T},
$$

where $J_{m, l}$ is an element of $\mathbf{J}$ in the $m$ th row and the lth column. For example:

$$
\mathbf{J}_{[2]}=\left[\begin{array}{cccc}
1 & 1 & 1 & 1 \\
1 & i & -i & -1 \\
1 & -i & i & -1 \\
1 & -1 & -1 & 1
\end{array}\right],
$$

is a Jacket matrix; thus, $\mathbf{J}_{[2]}^{+}$can be easily calculated by:

$$
\mathbf{J}_{[2]}^{+}=\frac{1}{4}\left[\begin{array}{cccc}
1 & 1 & 1 & 1 \\
1 & -i & i & -1 \\
1 & i & -i & -1 \\
1 & -1 & -1 & 1
\end{array}\right] .
$$

Furthermore, according to the Sylvester construction in terms of Kronecker product, the higher order of Jacket matrix can be recursively expressed by:

$$
\mathbf{J}_{[o d r]}=\mathbf{J}_{[o d r-1]} \otimes \mathbf{J}_{[1]},
$$

where $[o d r]=2^{o d r}$ denotes the matrix order.

\subsection{Single-band JS-DMT modem}

The block diagram describing the proposed single-band JS-DMT transceiver is illustrated in Figure 2 where the input sequence is transformed by the Jacket matrix before the IFFT. Compared to the Conventional DMT (C-DMT), an extra JS precoding stage and an extra inverse JS module emerged at the transmitter and the receiver, respectively.

Let $\mathbf{X}$ be the original input vector and $\mathbf{C}=$ $[\mathbf{X}, \mathcal{Z}(\mathbf{X})]^{T}$ follow Hermitian symmetry property. Assuming that $\mathbf{J}$ signifies an $N \times N$ Jacket matrix, the new transformed vector is given by:

$$
\mathbf{S}=\mathbf{J X}
$$

$\mathbf{C}_{\text {new }}=[\mathbf{S}, \mathcal{Z}(\mathbf{S})]^{T}$ is fed into IFFT module to yield the new transmitted signals. Therefore, the aperiodic $\mathrm{ACF}$ of $\mathbf{C}$ and $\mathbf{C}_{\text {new }}$ which satisfies:

$$
\xi_{\mathrm{C}_{\text {new }}}<\xi_{\mathrm{C}} .
$$

A detailed proof is given in the Appendix. According to Eq. (11), we can find that the PAPR of the new transmitted signals will be reduced efficiently.

\subsection{Multi-band JS-DMT modem}

In fact, if the used bandwidth of DMT exceeds the optimum range, the performance of JS-DMT is sensitive to non-linearity distortion and, thus, requires a robust equalizer at the receiver, which will increase the complexity of the whole system. In addition, the PAPR may be rapidly modified so that the low PAPR advantage at the transmitter will be negated and the PAPR reduction might be ineffective. Therefore, an improved MB-JS-DMT scheme with split symbolspread blocks is proposed to strengthen the robustness against fiber nonlinearity. Figure 3 demonstrates the conceptual diagram of MB-JS-DMT modulator with sub-band mapping. The entire spectrum of DMT is split into $L$ bands with $M$ sub-carriers per sub-band, which can be assigned with different modulation orders based on the sub-channel SNR.

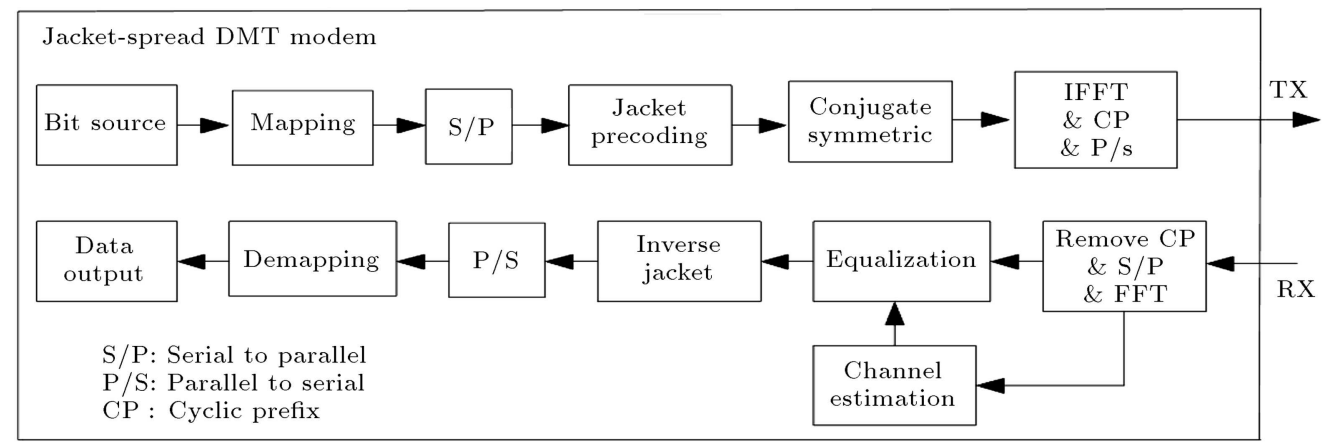

Figure 2. The diagram of DMT modem based on Jacket matrix spreading. 


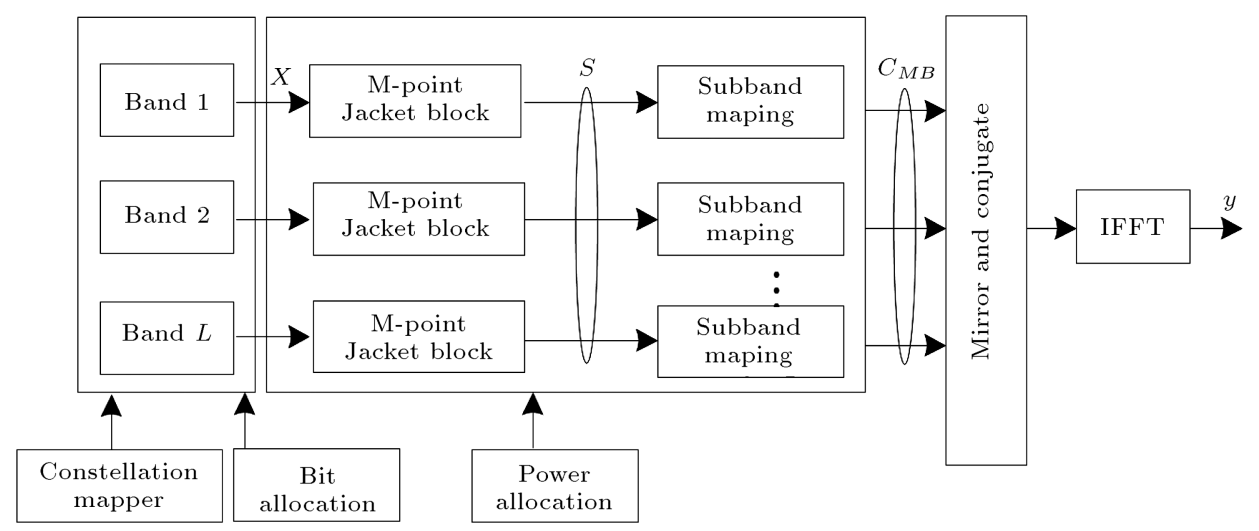

Figure 3. Block diagram of an MB-JS-DMT modulator.

Assuming that there are $N=M \cdot L$ sub-carriers, $X_{m}^{l}(l=0, \cdots, L-1, m=0, \cdots, M-1)$ denotes the $(m+1)$ th data symbol in the $(l+1)$ th band. Contrary to the single-band JS-DMT, original symbol $X_{m}^{l}$ is precoded by an $M \times M$ Jacket matrix firstly, and a new symbols is generated by:

$$
S_{k^{\prime}}^{l}=\sum_{m=0}^{M-1} X_{m}^{l} J_{m, k^{\prime}}, \quad k^{\prime}=0, \cdots, M-1 .
$$

$S_{k^{\prime}}^{l}$ is mapped onto:

$$
\begin{aligned}
\mathbf{C}_{M B}=\{ & \underbrace{C^{0}(0), \cdots}_{1 \mathrm{th}}, \underbrace{C^{1}(M), \cdots}_{2 \mathrm{th}}, \cdots, \\
& \underbrace{C^{L-1}((L-1) M), \cdots}_{L \mathrm{th}}\},
\end{aligned}
$$

where $C^{l}(k) \in \mathbf{C}_{M B}$ is the complex value according to the sub-band constellation mapping, expressed as follows:

$$
C^{l}(k)=\left\{\begin{array}{ll}
S_{k^{\prime}}^{l}, & k=k^{\prime}+M \cdot l \\
0, & \text { others }
\end{array} \quad k=0, \cdots, N-1 .\right.
$$

In addition, the input vector is obtained after Hermitian symmetry and fed to the IFFT block to generate the time-domain MB-JS-DMT signals $\mathbf{y}$, represented as follows:

$$
\mathbf{y}=\operatorname{IFFT}\{\mathbf{C}_{M B}, \underbrace{0, \cdots, 0}_{2\left(L_{0}-1\right) N}, \mathcal{Z}\left(\mathbf{C}_{M B}\right)\} .
$$

The demodulation process, designed as in Figure 4, is the reverse of Eqs. (18)-(20). Additionally, a simple zero forcing equalizer and a channel estimator are also adopted on the receiver side.

\section{Results and discussions}

To evaluate the performance of these proposed transmission schemes over IM/DD SI-POF link, extensive theoretical simulations and experimental tests are conducted. The setup diagram, based on offline processing in Matlab/Simulink, is depicted in Figure 5. The lowcost commercially available components, such as Resonant Cavity Light-Emitting Diodes (RC-LED) and PIN diode, with a Trans-Impedance Amplifier (TIA) are used. A standard $50 \mathrm{~m}$ Polymethyl Metacrylate (PMMA) SI-POF (150 dB/km@650 nm) is employed.

DMT signals are pre-generated by computer and stored in the Arbitrary Waveform Generator (AWG) memory. The analogue electrical signal of the AWG output is then used to drive a $650 \mathrm{~nm}$ RC-LED with a $10 \mathrm{~mA}$ bias current. After standard $50 \mathrm{~m}$ SI-POF transmission, the received optical signal is detected

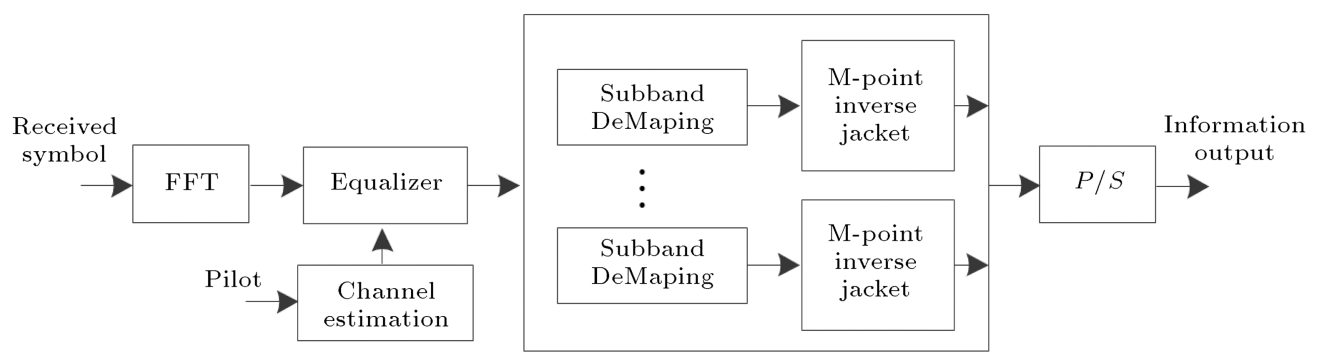

Figure 4. Block diagram of an MB-JS-DMT receiver. 


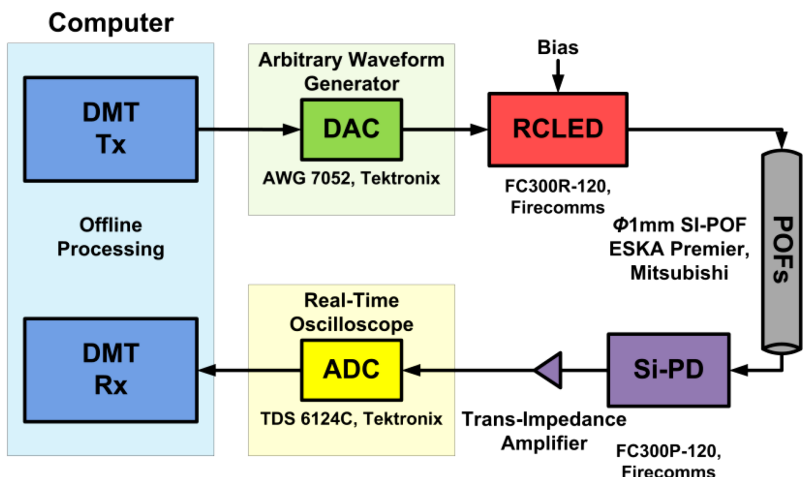

Figure 5. The experimental setup with offline processing.

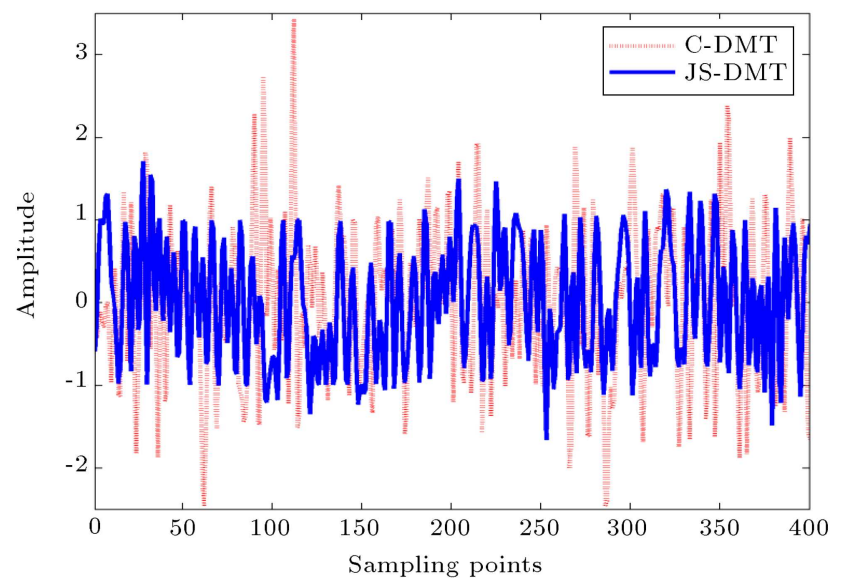

Figure 6. Amplitude comparisons between JS-DMT and C-DMT.

Table 1. The modulation parameters.

\begin{tabular}{lc}
\hline \multicolumn{1}{c}{ DMT } & Parameters \\
\hline Sampling frequency $(\mathrm{GHz})$ & 1 \\
IFFT/FFT & 2048 \\
Subcarrier spacing $(\mathrm{MHz})$ & 0.488 \\
Subcarrier number, $N$ & $1 \sim 1024$ \\
Cyclic Prefix (CP) ratio & $1 / 64$ \\
\hline
\end{tabular}

by a PIN diode and captured by ADC components. Finally, the digital signal processing is implemented offline in Matlab. The DMT modulation parameters are depicted in Table 1. According to the received probing symbols, the estimated channel information is obtained and the normalized noise PSD is measured around $-(106 \sim 114) \mathrm{dB} / \mathrm{Hz}$.

\subsection{Single-band DMT}

To assess the PAPR reduction in a single-band JSDMT modem, $N=512$ in Table 1 is selected. In our experiment, 10000 DMT symbols are randomly generated by a computer. Figure 6 shows the comparison result of amplitudes of JS-DMT and C-DMT. It is clearly manifested that the energy distribution of JS-DMT becomes more uniform and the large ampli-

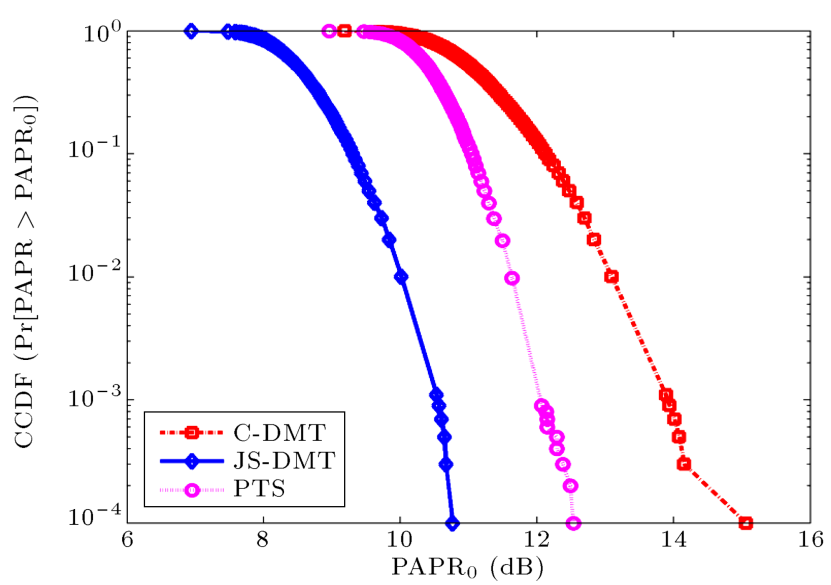

Figure 7. The CCDF comparisons among JS-DMT, C-DMT, and PTS.

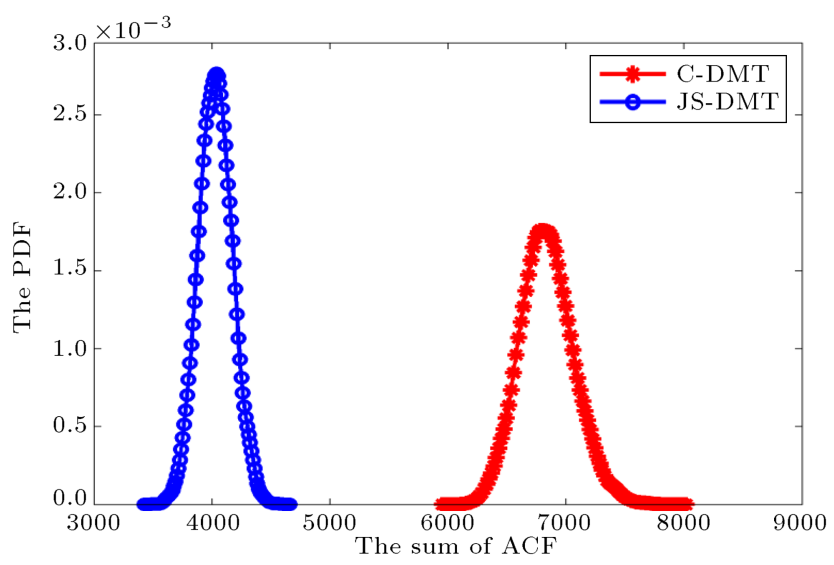

Figure 8. PDF comparisons between JS-DMT and $\mathrm{C}-\mathrm{DMT}$, in terms of $\xi_{\mathbf{C}_{\text {new }}}$ and $\xi_{\mathbf{C}}$.

tudes are compressed, resulting in a significant PAPR reduction. Figure 7 shows the CCDF performance of the JS-DMT. In addition, the well-known PTS is also implemented [23]. The parameters of PTS are chosen as follows: $U=16, V=4$, and $W=16(U, V$, and $W$ denote the number of modified candidate sequences, disjoint sub-vectors, and phase angles, respectively). Obviously, the probability of the PAPR in C-DMT, being larger than $14 \mathrm{~dB}$, is nearly $10^{-4}$. After JS precoding, the JS-DMT owns a much lower PAPR and almost $4 \mathrm{~dB}$ improvement is achieved. The PAPR performance of PTS is better than that of C-DMT, yet inferior to that of JS-DMT. However, the side information of PTS must be accurately known by the receiver; otherwise, the overall BER performance will be degraded. Furthermore, searching for optimal phase factors of PTS will cost more hardware resources and have higher computational complexity.

The ranges of $\left[\min \xi_{\mathbf{C}}, \max \xi_{\mathbf{C}}\right]$ and $\left[\min \xi_{\mathbf{C}_{n e w}}\right.$, $\left.\max \xi_{\mathbf{C}_{\text {new }}}\right]$ are divided into 99 equidistant intervals. Then, Probability Density Functions (PDFs) of CDMT and JS-DMT, with respect to $\xi_{\mathrm{C}}$ and $\xi_{\mathrm{C}_{\mathrm{new}}}$, are calculated and exhibited in Figure 8. The curves' 


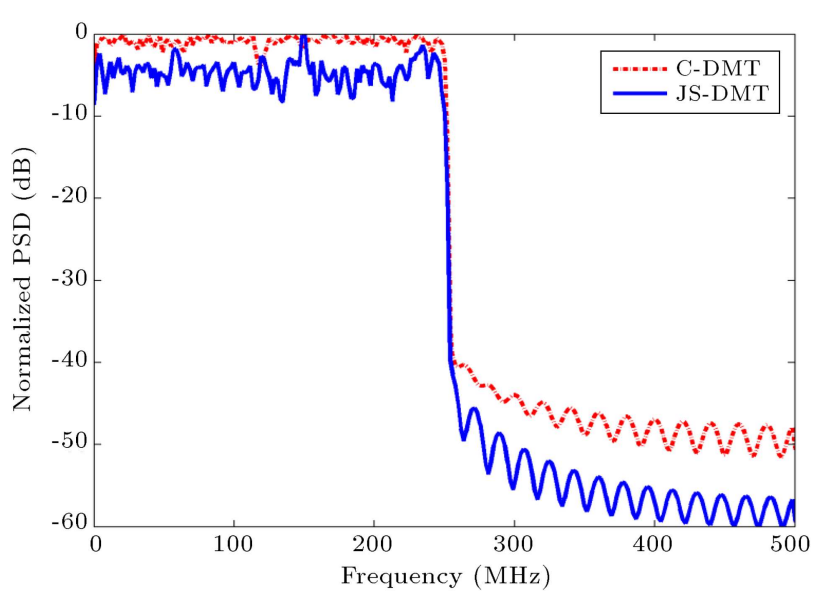

Figure 9. The PSD comparisons between JS-DMT and C-DMT.

shapes of PDF are alike, and $\xi$ has an approximate Gaussian distribution. $\xi_{\mathrm{C}_{\text {new }}}$ and $\mathbb{E}\left\{\xi_{\mathrm{C}_{\text {new }}}\right\}$ become smaller, implying that more PAPR reduction in JSDMT is achieved. Figure 9 depicts the comparison of the PSDs' performances. According to the figure, the rectangular-like and very sharp characteristics of DMT power spectrum remain retained and the sideband levels are also reduced, indicating that JS transformation does not damage the signal's PSD and the spectral efficiency.

\subsection{Multi-band JS-DMT}

Before investigating the MB-JS-DMT performance, sub-band number, $L$, which might affect the transmission performance, should be considered firstly to adopt the optimal used bandwidth. The performances of these four schemes, such as C-DMT, JS-DMT, 4band-JS-DMT (abbreviated as '4-band'), and 8-bandJS-DMT (abbreviated as '8-band'), are compared. For a fair comparison, all four schemes own the fixed input power and the same modulation parameters, which are depicted in Table 1. In addition, the BER target is set to $1 \times 10^{-3}$ which is convenient for reception with Forward Error Correction (FEC). Sub-carrier number $N$ varies from 1 to 1024 with the fixed sub-carrier spacing $\Delta f=0.488 \mathrm{MHz}$. Besides, the simplest Chow algorithm [24] is also employed due to its practicality and effectiveness in POF transmission.

Figure 10 shows CCDF performances of these four schemes. The C-DMT owns the highest PAPR, whereas the JS-DMT obtains the lowest. Considering MB-JS-DMT, the less the band split is, the lower the PAPR will be. Figure 11 shows the total achievable transmission rate of each system with respect to different used bandwidths. Accordingly, each scheme can reach the maximal transmission rate with its optimal used bandwidth. However, the transmission rate of JSDMT is sensitive to the bandwidth and its optimal range is limited only form $150 \mathrm{MHz}$ to $200 \mathrm{MHz}$,

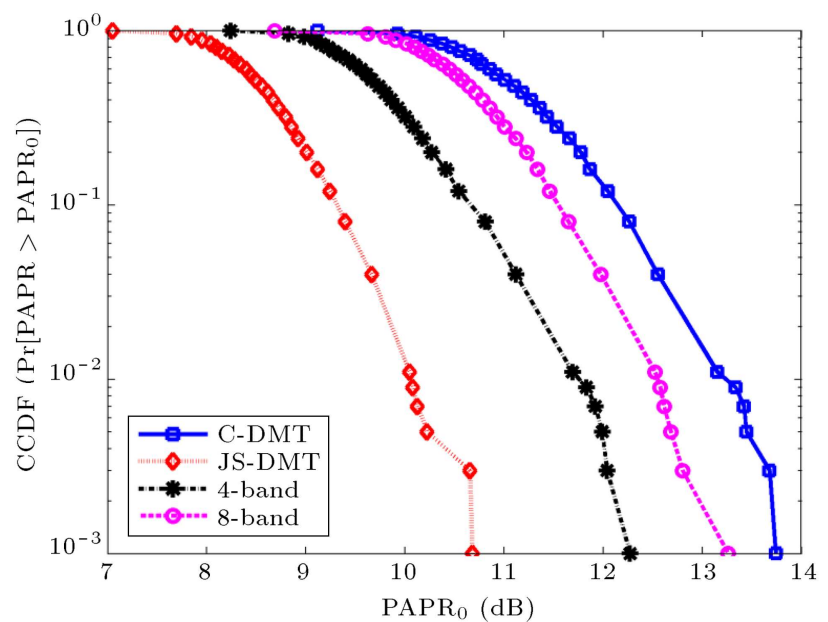

Figure 10. The PAPR performance of different schemes.

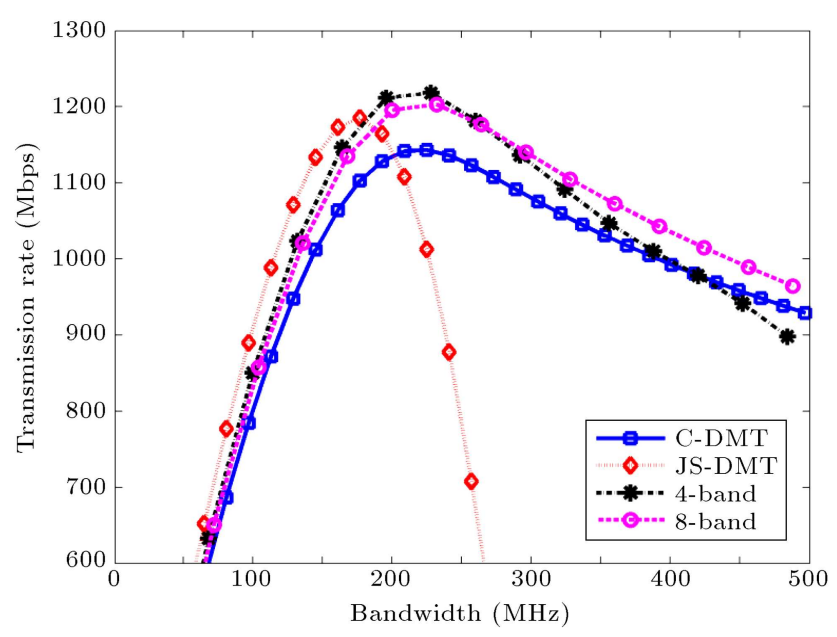

Figure 11. Transmission rate of different schemes.

implying that the capacity of JS-DMT will be severely degraded once the used bandwidth beyond this optimal range. However, fortunately, based on the figure, the MB-JS-DMT scheme owns the robustness ability against fiber nonlinearity, and their optimal bandwidth range is large. In addition, 4-band and 8-band achieve similar data rate and optimal used bandwidth. In order to make a good trade-off between transmission rate and PAPR performance, 4-band is usually chosen in the MB-JS-DMT since the efficient PAPR reduction can benefit from the SNR gains of the system.

\subsection{BER performance}

The BER performances of JS-DMT, MB-JS-DMT ( $L=$ 4), clipping, and PTS are investigated in this section. The DMT signal of these four schemes owns the same input power and the same modulation parameters, which are depicted in Table 1. The Chow algorithm [24] is adopted with the objective BER of $1 \times 10^{-3}$; in addition, the used sub-carrier of $N=512$ and the desired transmission rate of $1.2 \mathrm{Gbps}$ are selected. 


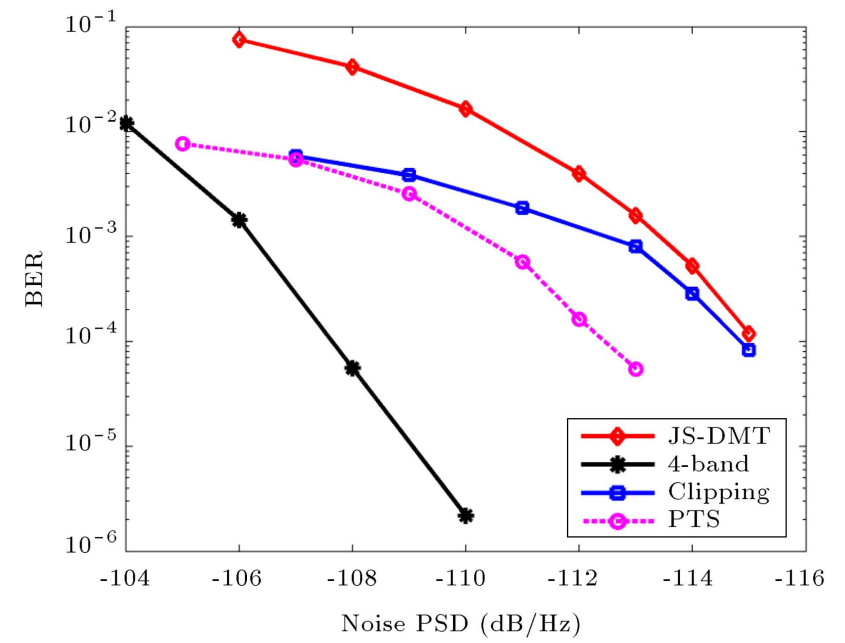

Figure 12. BER performance of different schemes.

After IFFT computations, the used bandwidths of DMT symbols are measured around $250 \mathrm{MHz}$. The crest factor (CR) of clipping is chosen by the typical value 2.5. Figure 12 manifests the measured noise PSD against BER performance for various transmission schemes.

The figure clearly indicates that JS-DMT offers the worst BER performance than the other schemes, because the bandwidth of $250 \mathrm{MHz}$ is beyond its optimum range. Clipping scheme is the simplest way to reduce the PAPR, whereas the total performance is limited by CR; besides, a higher PAPR reduction leads to a higher BER. PTS owns better BER performance than clipping; however, the overall BER is limited by side information detection. In the presence of side information detection failure, the demodulator cannot recover the original signal correctly; then, the BER performance is degraded. Moreover, multiple IFFT computations are required, and many operations of complex addition and multiplication have to be performed, which would cost much higher hardware resources.

Comparing the clipping and PTS at BER of $10^{-4}$, nearly $6.6 \mathrm{~dB}$ and $4.7 \mathrm{~dB}$ of power improvements are achieved by the proposed MB-JS-DMT schemes, respectively. JS precoding is the linear transformation without signal distortion at the transmitter, and the better BER performance is then obtained. In addition, only a very simple inverse Jacket matrix is employed for decoding and no side information is required in the proposed MB-JS-DMT scheme. What's more, not only did they have much lower computational complexity, but also they own the best BER performance and offer a better PAPR reduction, which is a very suitable trade-off.

In summary, the results demonstrate that MB-JSDMT are kinds of potential solution for the PAPR reduction and can be easily applied to the DMT modem, which is beneficial to high-speed POF transmissions based on the performance investigation of the CCDF, $\mathrm{BER}$, data rate, and PSD.

\section{Conclusions}

This paper discussed and investigated the PAPR problem in DMT modulator of the SI-POF system. The Jacket matrix spreading technique was introduced, and the novel modulation schemes, in terms of JS-DMT and MB-JS-DMT, were proposed for the PAPR reduction. By means of the new methodologies, the DMT signal with lower PAPR was produced. Extensive results and discussions manifested that JS precoding could reduce the PAPR efficiently while having no adverse impacts on PSD and BER performances. In addition, the MBJS-DMT scheme was more efficient for non-linearity mitigation by investigating the relationship between the transmission rates and used bandwidth. Both theoretical and experimental results demonstrated that the proposed JS modem was able to offer a favorable trade-off among the PAPR reduction, BER, data rate, and spectral efficiency, presenting a fact that the novel transmission schemes are practical and effective for future POF communications.

\section{Acknowledgments}

This work is supported in part by the Qingdao Postdoctoral Applied Research Project.

The authors would like to express gratitude to Prof. Wu, Dr. Peng, and Dr. Chen for their constructive suggestions and assistance to this article. We thank Dr. Peng for the assistance with equipment.

\section{References}

1. Okonkwo, C.M., Tangdiongga, E., Yang, H., Visani, D., Loquai, S., Kruglov, R., and Gaudino, R.C. "Recent results from the EU POF-PLUS project: Multigigabit transmission over $1 \mathrm{~mm}$ core diameter plastic optical fibers", J. Lightwave Technol., 29(2), pp. 186193 (2011).

2. Nespola, A., Abrate, S., Gaudino, R., Zerna, C., Offenbeck, B., and Weber, N. "High-speed communications over polymer optical fibers for in-building cabling and home networking", IEEE Photonics J., 2(3), pp. 347358 (2010).

3. Randel, S., Breyer, F., Lee, S.C., and Walewski, J.W. "Advanced modulation schemes for short-range optical communications", IEEE J. Sel. Top. Quant, 16(5), pp. 1280-1289 (2010).

4. Peng, L., Hélard, M., and Haese, S. "On bit-loading for discrete multi-tone transmission over short range POF systems", J. Lightwave Technol., 31(24), pp. 41554165 (2013). 
5. Miao, P., Wu, L., Chen, P., and Wang, X. "RCLED optimization and nonlinearity compensation in a polymer optical fiber DMT system", Appl. Sci., 6(9), p. 260 (2016).

6. Nunes, R.B., Helder, R.D.O., Segatto, M.E., and Silva, J.A. "Experimental validation of a constant-envelope OFDM system for optical direct-detection", Optical Fiber Technol., 20(3), pp. 303-307 (2014).

7. Tang, Y., Shieh, W., and Krongold, B.S. "DFT-spread OFDM for fiber nonlinearity mitigation", IEEE Photonics Technol. Lett., 22(16), pp. 1250-1252 (2010).

8. Lee, S.J., Breyer, F., Randel, S., Gaudino, R., Bosco, G., Bluschke, A., and Koonen, A.M. "Discrete multitone modulation for maximizing transmission rate in step-index plastic optical fibers", J. Lightwave Technol., 27(11), pp. 1503-1513 (2009).

9. Karabetsos, S., Pikasis, E., Nikas, T., Nassiopoulos, A., and Syvridis, D. "DFT-spread DMT modulation for $1-\mathrm{Gb} / \mathrm{s}$ transmission rate over $100 \mathrm{~m}$ of $1-\mathrm{mm}$ SIPOF", IEEE Photonic. Tech. Lett., 24(9), pp. 836-838 (2012).

10. Yu, H., Chen, M., and Wei, G. "Distribution of PAR in DMT systems", Electron. Lett., 39(10), pp. 799-801 (2003).

11. Lim, D.W., Heo, S.J., and No, J.S. "An overview of peak-to-average power ratio reduction schemes for OFDM signals", J. Commun. Netw. KOR., 11(3), pp. 229-239 (2009).

12. Jiang, T. and Wu, Y. "An overview: Peak-to-average power ratio reduction techniques for OFDM signals", IEEE Trans. Broadcast., 54(2), pp. 257-268 (2008).

13. Nadal, L., Moreolo, M.S., Fàbrega, J.M., and Junyent, G. "Low complexity PAPR reduction techniques for clipping and quantization noise mitigation in directdetection O-OFDM systems", Optical Fiber Technol., 20(3), pp. 208-216 (2014).

14. Armstrong, J. "Peak-to-average power reduction for OFDM by repeated clipping and frequency domain filtering", Electron. Lett., 38(5), pp. 246-247 (2002).

15. Jiang, T., Yao, W., Guo, P., Song, Y., and Qu, D. "Two novel nonlinear companding schemes with iterative receiver to reduce PAPR in multi-carrier modulation systems", IEEE Trans. Broadcast., 52(2), pp. 268-273 (2006).

16. Wang, C.L. and Ouyang, Y. "Low-complexity selected mapping schemes for peak-to-average power ratio reduction in OFDM systems", IEEE Trans. Signal Proces., 53(12), pp. 4652-4660 (2005).

17. Alsusa, E. and Yang, L. "Redundancy-free and BERmaintained selective mapping with partial phase- randomisingsequences for peak-to-average power ratio reduction in OFDM systems", IET Commun., 2(1), pp. 66-74 (2008).

18. Lee, M.H., Rajan, B.S., and Park, J.Y. "A generalized reverse Jacket transform", IEEE Trans. Circuits Syst. II, Analog Digit. Signal Process., 48(7), pp. 684-690 (2001).

19. Chen, X.H. and Lee, M.H. "A new spread sequence based on Jacket transform", J. South-Central University for Nationalities (Nat. Sci. Edn.), 22(3), pp. 32-35 (2003).

20. Wang, Z.P., Xiao, J.N., Li, F., and Chen, L. "Hadamard precoding for PAPR reduction in optical direct detection OFDM systems", Optoelectronics Lett., 7(5), pp. 363-366 (2011).

21. Zhu, X., Zhu, G., and Jiang, T. "Reducing the peakto-average power ratio using unitary matrix transformation", IET Commun., 3(2), pp. 161-171 (2009).

22. Jiang, T., Guizani, M., Chen, H.H., Xiang, W., and $\mathrm{Wu}, \mathrm{Y}$. "Derivation of PAPR distribution for OFDM wireless systems based on extreme value theory", IEEE Trans. Wireless Commun., 7(4), pp. 1298-1305 (2008).

23. Lu, G., Wu, P., and Carlemalm-Logothetis, C. "PAPR reduction for real baseband OFDM signals", 8th Int. Conf. on, Signal Processing, IEEE, Beijing (2006).

24. Chow, P.S., Cioffi, J.M., and Bingham, J.A. "A practical discrete multitone transceiver loading algorithm for data transmission over spectrally shaped channels", IEEE Trans. Commun., 43(234), pp. 773-775 (1995).

\section{Appendix}

$X_{u}$ is an independent and identically distributed variable with zero mean and variance $\sigma^{2} ; S_{u}$ is a mutually independent variable and its expectation is:

$$
\mathbb{E}\left\{S_{u}\right\}=\mathbb{E}\left\{J_{u} \mathbf{X}\right\}=J_{u} \mathbb{E}\{\mathbf{X}\}=0,
$$

where $J_{u}$ denotes the $u$ th row of $\mathbf{J}$. The variance of $\mathbf{S}$ can be calculated as follows:

$$
\sigma_{\mathbf{S}}^{2}=\mathbb{E}\left\{\mathbf{S S}^{H}\right\}=\mathbf{J} \mathbb{E}\left\{\mathbf{X} \mathbf{X}^{H}\right\} \mathbf{J}^{H}=\sigma^{2} \mathbf{I}_{\mathbf{N}},
$$

where $\mathbf{I}_{\mathbf{N}}$ is the identity matrix. $\xi_{\mathbf{S}}$ and $\xi_{\mathbf{X}}$ are random variables; thus, we have:

$$
\begin{aligned}
& \mathbb{E}\left\{\xi_{\mathbf{S}}\right\}=\mathbb{E}\left\{\sum_{q=1}^{N-1}\left|\rho_{\mathbf{S}}(q)\right|\right\}=\sum_{q=1}^{N-1} \mathbb{E}\left\{\left|\rho_{\mathbf{S}}(q)\right|\right\}, \\
& \mathbb{E}\left\{\xi_{\mathbf{X}}\right\}=\sum_{q=1}^{N-1} \mathbb{E}\left\{\left|\rho_{\mathbf{X}}(q)\right|\right\} .
\end{aligned}
$$

For $q=N-\tau(1 \leq \tau \leq N)$, we can calculate:

$$
\begin{aligned}
& \mathbb{E}\left\{\left|\rho_{\mathbf{S}}(q)\right|^{2}\right\} \\
& =\mathbb{E}\left\{\left(\sum_{k=0}^{\tau-1} S_{k+N-\tau} S_{k}^{*}\right)\left(\sum_{k=0}^{\tau-1} S_{k+N-\tau} S_{k}^{*}\right)^{*}\right\} .
\end{aligned}
$$


Owing to $S_{N-1}, \cdots, S_{N-\tau}$, and $S_{0}^{*}, \cdots, S_{\tau-1}^{*}$ are mutually independent, and Eq. (A.5) can be simplified as follows:

$$
\begin{aligned}
\mathbb{E}\left\{\left|\rho_{\mathrm{S}}(q)\right|^{2}\right\}= & \mathbb{E}\left\{\left|S_{N-\tau} S_{0}^{*}\right|^{2}\right\}+\mathbb{E}\left\{\left|S_{N-\tau+1} S_{1}^{*}\right|^{2}\right\} \\
& +\cdots+\mathbb{E}\left\{\left|S_{N-1} S_{\tau-1}^{*}\right|^{2}\right\} .
\end{aligned}
$$

Accordingly, the similar expression of $\mathbb{E}\left\{\left|\rho_{\mathbf{X}}(q)\right|^{2}\right\}$ can be derived as follows:

$$
\begin{aligned}
\mathbb{E}\left\{\left|\rho_{\mathbf{X}}(q)\right|^{2}\right\}= & \mathbb{E}\left\{\left|X_{N-\tau} X_{0}^{*}\right|^{2}\right\} \\
& +\mathbb{E}\left\{\left|X_{N-\tau+1} X_{1}^{*}\right|^{2}\right\} \\
& +\cdots+\mathbb{E}\left\{\left|X_{N-1} X_{\tau-1}^{*}\right|^{2}\right\}
\end{aligned}
$$

For the special case $\tau=1$, on the one hand, we have:

$$
\begin{aligned}
& \mathbb{E}\left\{\left|\rho_{\mathbf{S}}(N-1)\right|\right\}=\mathbb{E}\left\{\left|S_{N-1} S_{0}^{*}\right|\right\} \\
& \quad=\mathbb{E}\left\{\left|S_{N-1}\right| \times\left|S_{0}\right|\right\}=\mathbb{E}\left\{\left|J_{N-1} \mathbf{X}\right| \times\left|J_{0} \mathbf{X}\right|\right\} .
\end{aligned}
$$

By the Cauchy-Schwartz inequality, Eq. (A.8) can be further arrived at:

$$
\begin{aligned}
& \mathbb{E}\left\{\left|J_{N-1} \mathbf{X}\right| \times\left|J_{0} \mathbf{X}\right|\right\} \\
& \quad<\sqrt{\mathbb{E}\left\{\left|J_{N-1} \mathbf{X}\right|^{2}\right\} \times \mathbb{E}\left\{\left|J_{0} \mathbf{X}\right|^{2}\right\}} \\
& =\sqrt{\left(J_{N-1} \mathbb{E}\left\{\mathbf{X X}^{H}\right\} J_{N-1}^{H}\right)\left(J_{0} \mathbb{E}\left\{\mathbf{X X}^{H}\right\} J_{0}^{H}\right)} \\
& =\sigma^{2} .
\end{aligned}
$$

On the other hand, we have:

$$
\mathbb{E}\left\{\left|\rho_{\mathbf{X}}(N-1)\right|\right\}=\mathbb{E}\left\{\left|X_{N-1}\right| \times\left|X_{0}\right|\right\}=\sigma^{2} .
$$

Therefore, we can obtain:

$$
\mathbb{E}\left\{\left|S_{N-1} S_{0}^{*}\right|\right\}<\mathbb{E}\left\{\left|X_{N-1} X_{0}^{*}\right|\right\} .
$$

Similarly, for $\tau=2$, we will arrive at:

$$
\begin{aligned}
& \mathbb{E}\left\{\left|S_{N-2} S_{0}^{*}\right|\right\}+\mathbb{E}\left\{\left|S_{N-1} S_{1}^{*}\right|\right\} \\
& \quad<\mathbb{E}\left\{\left|S_{N-2} S_{0}^{*}\right|\right\} \\
& \quad+\mathbb{E}\left\{\left|S_{N-1} S_{1}^{*}\right|\right\} .
\end{aligned}
$$

For $\tau=3,4, \cdots, N$, the similar inequality relationships as Eqs. (A.11) and (A.12) are achieved. Thus, we can deduce that:

$$
\mathbb{E}\left\{\left|\rho_{\mathbf{S}}(q)\right|^{2}\right\}<\mathbb{E}\left\{\left|\rho_{\mathbf{X}}(q)\right|^{2}\right\}
$$

and:

$$
\mathbb{E}\left\{\xi_{\mathbf{s}}\right\}<\mathbb{E}\left\{\xi_{\mathbf{X}}\right\}
$$

Finally, the mean values of $\xi_{\mathbf{C}_{\text {new }}}$ and $\xi_{\mathbf{C}}$, respecting $\mathbf{C}_{\text {new }}=[\mathbf{S}, \mathcal{Z}(\mathbf{S})]^{T}$ and $\mathbf{C}=[\mathbf{X}, \mathcal{Z}(\mathbf{X})]^{T}$, have the following relation:

$$
\mathbb{E}\left\{\xi_{\mathbf{C}_{\text {new }}}\right\}<\mathbb{E}\left\{\xi_{\mathrm{C}}\right\} .
$$

As $N$ is large enough, $\xi_{\mathrm{C}_{\text {new }}}$ approaches an approximate Gaussian distribution. The reduction of the mean value implies a lower $\xi_{\mathbf{C}_{\text {new }}}$. Therefore, it is inferred that the lower $\xi_{\mathbf{C}_{\text {new }}}$ is, the better the PAPR performance will be.

\section{Biographies}

Pu Miao received his BS and MS degrees from Zhen gzhou University and Henan University of Science and Technology in 2008 and 2011, respectively, and the $\mathrm{PhD}$ degree in Information and Communication Engineering from School of Information Science and Engineering, Southeast University, Nanjing, China in 2015. He is currently an Assistant Professor in the School of Electronic and Information Engineering, Qingdao University, Qingdao, China. His current research interests include signal processing, coding, channel estimation and multicarrier modulation techniques applied to wireless communications and optical communications.

Lenan Wu received the $\mathrm{PhD}$ degree in Signal and Information Processing from Southeast University, Nanjing, China, in 1997. He is currently a Full Professor in Institute of Multimedia Technology, School of Information Science and Engineering, Southeast University, Nanjing, China. He has authored or coauthored over 100 technical papers in major journals and conferences in the areas of signal processing and communications. His current research interests include the areas of wireless communications and corresponding signal processing, especially for high efficiency modulation technique.

Linning Peng received his BS and MS degrees in 2006 and 2010, respectively, from the School of Information Science and Engineering, Southeast University, China, and the PhD degree in 2014 from the Electronics and Telecommunications Institute of Rennes (IETR) laboratory at the National Institute of Applied Sciences (INSA) of Rennes, France. In 2014, he joined Southeast University where he is currently an Associate Professor in the School of Electronic and Information Engineering. His current research interests focus on physical layer security, optical communications, and indoor optical networking. 